\title{
Is primary total pancreatectomy in patients with high- risk pancreatic remnant justified and preferable to pancreaticoduodenectomy? - a matched-pairs analysis of 200 patients
}

\author{
Andreas Minh Luu ${ }^{1 \#}$, Bella Olchanetski ${ }^{1 \#}$, Torsten Herzog ${ }^{1}$, Andrea Tannapfel $^{2}$, Waldemar Uhl ${ }^{1}$, \\ Orlin Belyaev ${ }^{1 \wedge}$
}

${ }^{1}$ Department of Surgery, St. Josef Hospital, Ruhr University Bochum, Bochum, Germany; ${ }^{2}$ Institute of Pathology, Ruhr University Bochum, Bochum, Germany

Contributions: (I) Conception and design: O Belyaev, W Uhl, B Olchanetski, AM Luu; (II) Administrative support: W Uhl, A Tannapfel, O Belyaev; (III) Provision of study materials and patients: W Uhl, A Tannapfel, O Belyaev, T Herzog; (IV) Collection and assembly of data: B Olchanetski, O Belyaev, W Uhl, A Tannapfel, AM Luu, T Herzog; (V) Data analysis and interpretation: All authors; (VI) Manuscript writing: All authors; (VII) Final approval of manuscript: All authors.

\#These authors contributed equally to this work as co-first authors.

Correspondence to: Orlin Belyaev, MD, PhD. Department of Surgery, St. Josef Hospital, Ruhr University Bochum, Gudrunstr. 56, 44791 Bochum, Germany. Email: orlin.belyaev@klinikum-bochum.de.

Background: Total pancreatectomy (TP) eliminates the risk of postoperative pancreatic fistula (POPF) and its associated secondary complications. Hence, it may theoretically offer advantages over pancreaticoduodenectomy (PD) regarding early postoperative outcome of patients with high-risk pancreatic remnant.

Methods: Ninety-day mortality and morbidity of $100 \mathrm{TP} v s .100$ PD for pancreatic head lesions were retrospectively compared. Groups were matched for pancreatic texture, pancreatic duct size, final histology, age, gender and surgeon. Only patients at high risk for POPF due to soft pancreatic texture and small pancreatic duct $<3 \mathrm{~mm}$ were included.

Results: Preoperatively, the TP-group was characterized by poorer general condition, more comorbidities and more pronounced obesity than the PD-group. Postoperatively, overall morbidity was lower after TP (63\% vs. 88\%, $\mathrm{P}<0.001)$ due to less mild complications. Postpancreatectomy hemorrhage rate was lower after TP than after PD (2\% vs. 12\%, P=0.014). Duration of surgery, hospital stay, major morbidity (30\%) and mortality (7\% vs. $5 \%$ ) were the same. POPF was the most common complication after PD with $32 \%$. Emergency completion pancreatectomy was necessary in $10 \%$ of PD with a significantly higher mortality compared to elective TP (50\% vs. 7\%, P=0.001).

Conclusions: TP may reduce severe POPF-associated complications and prevent mortality related to emergency completion pancreatectomy in some elderly, obese and polymorbid patients with high-risk pancreatic remnant. Careful individual selection by an experienced pancreatic surgeon is mandatory.

Keywords! Pancreatectomy; pancreaticoduodenectomy; pancreatic fistula; morbidity; outcome

Submitted Aug 15, 2020. Accepted for publication Nov 11, 2020.

doi: 10.21037 /gs-20-670

View this article at: http://dx.doi.org/10.21037/gs-20-670

\footnotetext{
^ ORCID: 0000-0002-0595-2292.
} 


\section{Introduction}

Reconstruction after pancreaticoduodenectomy (PD) in the presence of soft pancreatic remnant and small-sized pancreatic duct is a challenge even to the experienced pancreatic surgeon. These features are recognized as major risk factors for postoperative pancreatic fistula (POPF), which itself remains the most relevant source of morbidity after PD $(1,2)$. Rates of clinically relevant POPF are well above $20 \%$ at high-volume centers, reaching even $40 \%$ in the subgroup of patients with high-risk pancreatic remnant (3-5). POPF has the potential to cause life-threatening secondary complications such as postpancreatectomy hemorrhage (PPH), intraabdominal abscess, and sepsis, leading to increased costs, prolonged hospital stay as well as to delayed chemotherapy in oncologic patients $(2,6)$.

As early as the 1950 s of the $20^{\text {th }}$ century the pioneers of pancreatic surgery hoped that total pancreatectomy (TP) may offer advantages over PD not only in terms of oncologic radicality but also by means of reducing mortality and morbidity (7-10). Despite promising short-term results and continuous reduction of mortality rates, TP was widely abandoned as a treatment option in the next decades because of its devastating long-term effects including brittle diabetes and total exocrine pancreatic insufficiency, which were difficult to manage in those days. At the same time advances in surgical technique and intensive care turned PD into a safe and standardized procedure with acceptably low mortality. However, neither advances in medical knowledge nor centralization of care had any success in reducing short-term major morbidity after $\mathrm{PD}$, mainly due to constant or even increasing rates of POPF $(1,4)$. Driven by the tremendous progress of insulin treatment and enzyme replacement as well as the evolution of surgical technique and benefits of centralization, the debate on the role of primary TP at the time of surgery for pancreatic head lesions has recently reawakened (11-14). The selective use of such "prophylactic" TP is of particular interest for the population of patients at a very high risk for severe POPF due to soft pancreatic texture and tiny pancreatic duct. Theoretically, mortality and major morbidity related to complications at the pancreatic remnant in the early postoperative period may be reduced by TP $(12,14)$.

The present study aimed to assess if primary "prophylactic" TP offered any advantages regarding early postoperative outcomes compared to conventional PD in a selected population of patients with high-risk pancreatic remnant.
The following article is presented in accordance with the STROBE reporting checklist (available at http://dx.doi. org/10.21037/gs-20-670).

\section{Methods}

\section{Patients}

This retrospective matched-pairs study was approved by the Ethics Committee of the Ruhr University Bochum (No. 17-6321) and conducted in accordance with the Helsinki Declaration as revised in 2013. Informed consent for surgery as well as for data collection and analysis was given by all patients.

Between January 2009 and December 2018, all patients who underwent primary elective TP for high-risk pancreatic remnant were retrospectively compared to a carefully matched same-sized group of patients who underwent PD. All included patients were characterized by a very soft pancreatic remnant and had a pancreatic duct smaller than $3 \mathrm{~mm}$. Pancreatic hardness was assessed by the surgeon and quantitatively confirmed by a Shore durometer, with values under $40 \mathrm{SU}$ indicating soft tissue (15). Pancreatic duct size was measured with a ruler at surgery and confirmed at final pathology. Patient history, clinical, pathological, radiological data and outcomes were prospectively recorded in the pancreas center data bank.

\section{Surgical technique and perioperative management}

All procedures were performed by four experienced pancreatic surgeons ( $>50$ pancreatic operations per year) according to a standardized surgical technique: Reconstruction after PD included an end-to-side, duct-tomucosa, double-layer pancreaticojejunostomy (PJ) using interrupted polydioxanone (PDS) 5-0 suture for the outer layer and interrupted polypropylene 5-0 suture for the inner layer. Neither sealants, nor stents were applied at the PJ. A single-layer end-to-side hepaticojejunostomy ( $\mathrm{HJ})$ using interrupted PDS 5-0 suture was performed. In patients with thin-walled and tiny hepatic ducts, PDS 6-0 was used and the $\mathrm{HJ}$ was splinted using an externally diverted T-tube at discretion of the surgeon. Antecolic duodenojejunostomy was performed $50 \mathrm{~cm}$ distal to $\mathrm{HJ}$ in a double-layer continuous PDS 5-0 suture technique. In patients without pylorus preservation a Braun enteroenterostomy was added to gastrojejunostomy. All patients received two intraabdominal soft silicone drains placed in the vicinity of 
the $\mathrm{HJ}$ and PJ, derived separately through the skin of the right and left middle abdomen. Perioperative octreotide was routinely administered. In TP the spleen was removed on discretion of the surgeon and except for the omitted PJ, reconstruction was the same as in PD. Patients with PD were monitored on the ICU over night and brought back to the peripheral ward the day after surgery. As a rule, patients with TP were monitored for 2-3 days on the ICU to allow better control of glucose levels and electrolytes in the early postoperative period.

\section{Postoperative outcome}

Delayed gastric emptying (DGE), POPF, PPH, bile and chyle leaks were defined according to the consensus definitions by the International Study Group on Pancreatic Surgery (16-20). Complications were classified according to the criteria of Clavien and Dindo, whereas grade III or greater were regarded as major complications (21). As the study focused on short-term surgical outcomes all events including mortality occurring within 90 days postoperatively or during the hospital stay, if longer, were considered for the assessment of results. Complete and detailed clinical and pathological reports were available for all patients.

\section{Statistical analysis}

Continuous variables are expressed as median (i.q.r.) and categorical variables are presented as absolute numbers and percentages. An independent samples two-tailed $t$-test (for normally distributed data) and Mann-Whitney U-test (for abnormally distributed data) were used to compare continuous variables between the study groups. The chisquared test was used for categorical variables. Events were considered statistically significant if $\mathrm{P}<0.05$. The analysis was performed with SPSS software release 22.0 (SPSS Inc., Chicago, Ill., USA).

\section{Results}

\section{Patients}

A total of $414 \mathrm{TP}$ were performed at our institution during the study period of 10 years. One hundred of these patients underwent TP mainly because the pancreatic remnant was found technically unsuitable for a safe anastomosis due to soft and friable pancreatic texture combined with smallsized pancreatic duct. The risk for postoperative life- threatening complications related to a risky pancreatic anastomosis was intraoperatively rated as unacceptably high by the surgeon and a TP instead of PD was performed at the time of index surgery. Other factors such as poor remnant perfusion, positive resection margin, acute postendoscopic retrograde cholangiopancreatography (ERCP) pancreatitis of the remnant at the time of surgery, preexistent insulin dependent diabetes mellitus or technical problems during creation of the pancreatic anastomosis may have played an additional role to favour the intraoperative choice of TP over PD. These $100 \mathrm{TP}$ were compared to a control group of $100 \mathrm{PD}$, which were selected from 777 PD performed during the study period, after careful 1:1 matching for final histology (benign vs. malignant), gender, age, surgical team, pancreatic texture and size of the main pancreatic duct.

\section{Characteristics of patients}

Patients in the TP group were in a poorer health condition and sicker than those with PD: in the TP group 39\% were classified as the American Society of Anesthesiologists (ASA) III-IV compared to $22 \%$ of $\mathrm{PD}(\mathrm{P}=0.003)$, mainly due to a higher rate of accompanying cardiovascular disease. Patients with TP were more obese, resulting in statistically higher calculated fistula risk scores compared to PD (Table 1).

\section{Surgical outcomes}

Duration of surgery and estimated blood loss didn't significantly differ between TP and PD (Table 2). ICU stay was longer after TP due to the standard protocol of postoperative management as already mentioned in the methods section. The total postoperative hospital stay after TP was not longer than after PD. Mortality and major morbidity rates after TP and PD were the same. Significantly more patients had an uneventful postoperative course after TP than after PD. Overall morbidity was less after TP due to a significantly lower incidence of minor postoperative complications ( $35 \%$ vs. $58 \%, \mathrm{P}<0.001$ ). Clinically relevant POPF occurred in $32 \%$ after PD. PPH was significantly less frequent after TP (2\% vs. $12 \%$, $\mathrm{P}=0.014)$. The reason for bleeding in 10 of $12 \mathrm{PPH}$ was arterial vessel erosion due to POPF grade C: affected vessels were the gastroduodenal artery $(n=2)$, the right hepatic artery $(n=2)$, the splenic artery $(n=2)$, the common hepatic artery $(n=1)$, the celiac trunk $(n=1)$, the superior mesenteric artery $(n=1)$, and the left gastric artery $(n=1)$. 
Table 1 Patient characteristics

\begin{tabular}{|c|c|c|c|}
\hline Characteristics & TP $(n=100)$ & $P D(n=100)$ & $\mathrm{P}$ value \\
\hline Gender (male:female) & $53: 47$ & $53: 47$ & Matched \\
\hline Soft pancreatic texture & 100 & 100 & Matched \\
\hline Durometric hardness (SU)* & 25 [15-30] & 25 [15-30] & Matched \\
\hline Pathology (benign:malignant) & 33:67 & $33: 67$ & Matched \\
\hline PDAC & 31 & 26 & \\
\hline Papillary carcinoma & 15 & 17 & \\
\hline Bile duct carcinoma & 14 & 16 & \\
\hline Benign cystic neoplasms ${ }^{\star \star}$ & 23 & 25 & \\
\hline Common bile duct size $(\mathrm{mm})^{\star}$ & $7[5-11]$ & $7[4-10]$ & 0.292 \\
\hline ASA class & & & 0.003 \\
\hline ASA I & 0 & 4 & \\
\hline ASA II & 61 & 74 & \\
\hline ASA III & 36 & 21 & \\
\hline ASA IV & 3 & 1 & \\
\hline $\mathrm{BMI}^{\star}\left(\mathrm{kg} / \mathrm{m}^{2}\right)$ & 28 [25-30] & 24 [21-28] & $<0.001$ \\
\hline Any comorbidities & 86 & 64 & $<0.001$ \\
\hline Cardiovascular disease & 75 & 49 & $<0.001$ \\
\hline Pulmonary disease & 18 & 17 & 0.853 \\
\hline Renal insufficiency & 6 & 4 & 0.479 \\
\hline Past surgical history & 71 & 62 & 0.146 \\
\hline Preoperative biliary stent & 39 & 36 & 0.661 \\
\hline Reconstruction (pp:classic) & $88: 12$ & $92: 8$ & 0.346 \\
\hline Splenectomy & 76 & n.a. & - \\
\hline
\end{tabular}

*, median and interquartile ranges represented if not otherwise specified; **, intraductal papillary mucinous neoplasm (IPMN), mucinous cystic neoplasm (MCN), serous cystic neoplasm $(\mathrm{SCN})$ and solid pseudopapillary neoplasm (SPN). TP, total pancreatectomy; PD, pancreaticoduodenectomy; SU, Shore units; PDAC, pancreatic ductal adenocarcinoma; PNEN, pancreatic neuroendocrine neoplasm; ASA, American Society of Anesthesiologists; BMI, body mass index; a-FRS, alternative Fistula Risk Score calculated online according to the formula of Mungroop et al. (22); pp, pylorus preserving; n.a., not applicable. 
Table 2 Perioperative outcomes

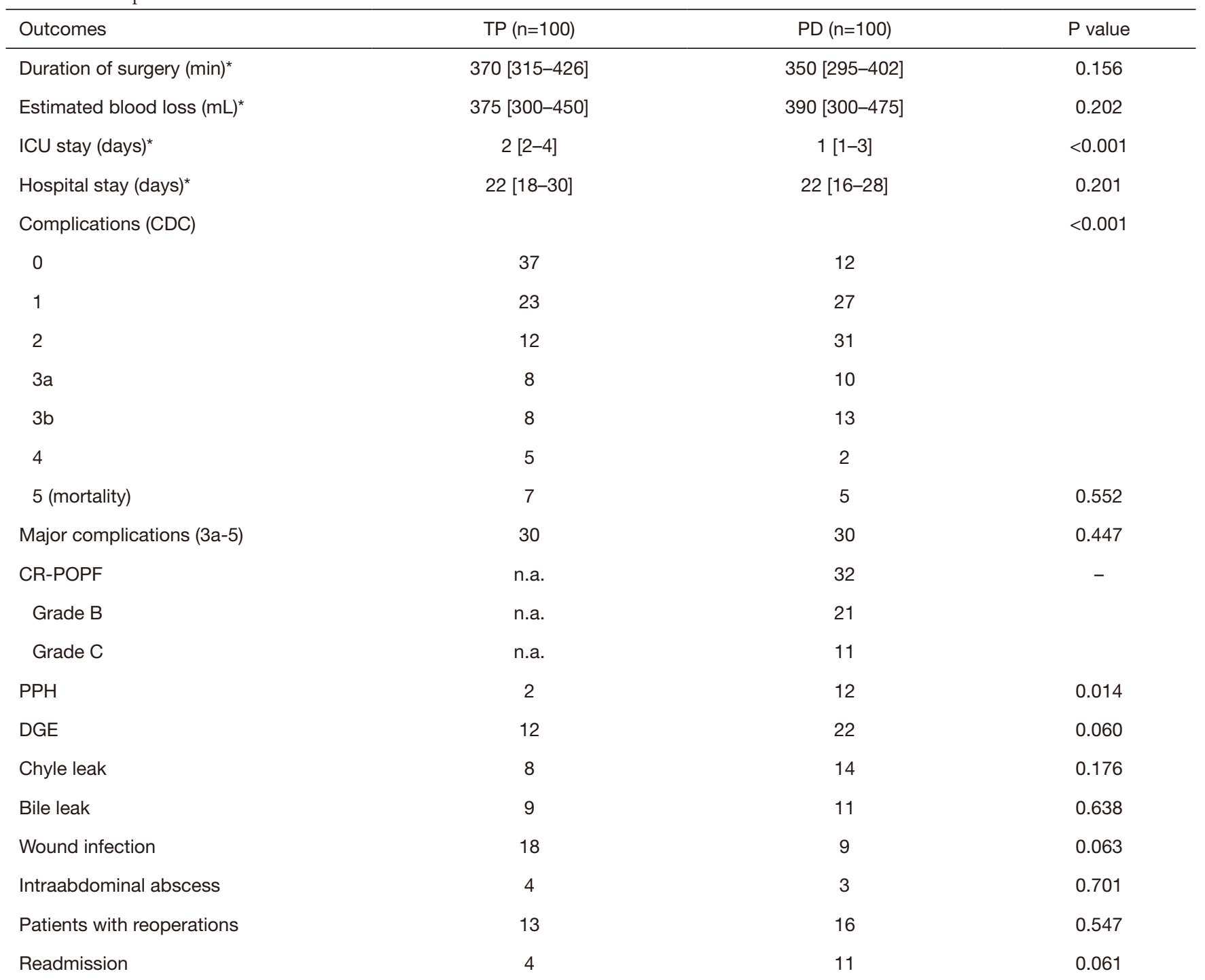

*, median and interquartile ranges represented if not otherwise specified. ICU, intensive care unit; CDC, Clavien-Dindo classification; CRPOPF, clinically relevant postoperative pancreatic fistula; PPH, postpancreatectomy hemorrhage; DGE, delayed gastric emptying; n.a., not applicable.

Two patients suffered PPH after TP and none of them was caused by a POPF: the first one had erosion bleeding of the right hepatic artery due to a bile leak and the second patient suffered a spleen hemorrhage after a spleen preserving TP. Both patients underwent emergency reoperations and recovered well.

\section{Reoperations}

Emergency revisional surgery was necessary in 13 patients after TP and 16 patients after PD (Table 3). Reoperations after PD were significantly more often associated with multiple revisional procedures $(\mathrm{P}=0.031)$. The most common indication for reoperation in the TP group was leakage of the HJ. The spectrum of indications for reoperations after PD was dominated by severe complications related to the pancreatic remnant. Eleven of 16 patients developed a severe leak at the PJ accompanied by an acute necrotizing postoperative pancreatitis of the pancreatic remnant in 6 cases and arterial erosion 
Table 3 Detailed comparison of emergency reoperations after TP and PD

\begin{tabular}{|c|c|c|c|}
\hline Variables & TP & PD & $P$ value \\
\hline Age (years) ${ }^{*}$ & 75 [74-76] & $60[53-77]$ & 0.086 \\
\hline Gender (m:f) & $8: 5$ & $9: 7$ & 1.000 \\
\hline Total number of reoperations & 17 & 26 & \\
\hline PJ leak/pancreatic necrosis & n.a. & 11 & \\
\hline $\mathrm{PPH}$ & 2 & 10 & \\
\hline HJ leak & 5 & 3 & \\
\hline GE leak & 2 & 0 & \\
\hline Thrombosis of hepatic artery & 1 & 0 & \\
\hline Completion pancreatectomy & n.a. & 10 & \\
\hline Postoperative day of reoperation* & $10[6-12]$ & 13 [6-22] & 0.218 \\
\hline Mortality after reoperation & $2 / 13(15 \%)$ & $5 / 16(31 \%)$ & 0.410 \\
\hline ICU stay $\left(\right.$ days) ${ }^{\star}$ & $6[3-26]$ & $6[4-17]$ & 0.566 \\
\hline Postoperative stay (days) ${ }^{*}$ & 38 [22-69] & $32[17-44]$ & 0.392 \\
\hline
\end{tabular}

*, median and interquartile ranges represented if not otherwise specified; ${ }^{\star \star}$, multiple complications per patient possible, i.e., anastomotic leak and PPH. TP, total pancreatectomy; PD, pancreaticoduodenectomy; PJ, pancreaticojejunostomy; PPH, postpancreatectomy hemorrhage; HJ, hepaticojejunostomy; GE, gastroenterostomy; ICU, intensive care unit; n.a., not applicable.

hemorrhage in 10 patients. Respectively, the most common emergent surgical procedure at reoperation in the PD group was the rescue completion pancreatectomy. Both ICU and total postoperative hospital stay were significantly longer for the subgroups of reoperated patients after TP and $\mathrm{PD}$ compared to the median values of the entire cohort of patients $(\mathrm{P}<0.001)$. The hospital stay and ICU stay of reoperated patients was comparable between the TP and PD groups.

\section{Mortality}

All lethal cases after PD occurred after emergency completion pancreatectomy for complications of the pancreatic remnant. Mortality after emergency completion pancreatectomy was significantly higher than mortality after elective prophylactic TP [50\% (5/10) vs. 7\% (7/100), $\mathrm{P}=0.001]$.

Mortality in the TP group had a different spectrum of sources. Only 2 of 7 lethal outcomes were associated with relevant surgical complications: one male underwent reoperation for early $\mathrm{HJ}$ leakage with peritonitis, followed by severe pneumonia and pulmonary embolism, and the second patient, an extremely obese female, underwent resection of the hepatic artery and reconstruction by a splenohepatic switch, but suffered an early and complete thrombosis of the artery and died of acute liver and multiorgan failure (MOF). The rest 5 deceased patients in the TP group died of pneumonia and progressive respiratory failure, accompanied by MOF in the terminal phase. All deceased patients in both groups underwent surgery for malignant disease and suffered one or more serious comorbidities: all 7 patients in the TP group had advanced COPD, and all 5 patients in the PD group had advanced cardiovascular disease. The median age of deceased patients was higher than the median of all 200 patients (74.5 vs. 68 years, $\mathrm{P}=0.052$ ). Median age and body mass index (BMI) of deceased patients after PD and TP 
didn't differ: 76 vs. 73 years, $\mathrm{P}=0.418$ and $28 \mathrm{~kg} / \mathrm{m}^{2} v s$. $26 \mathrm{~kg} / \mathrm{m}^{2}, \mathrm{P}=0.904$.

\section{Discussion}

Despite major developments in surgical technique and perioperative management, rates of POPF and $\mathrm{PPH}$ remain a constant and unsolved problem after PD and represent the most relevant triggers of mortality (1-5). After its introduction in 1942 by Rockey, TP was advocated by some pioneers of pancreatic surgery as prophylaxis of POPF and its associated morbidity and mortality, but abandoned later due to the lack of oncologic benefit and the detrimental effects of apancreatic state (7-10). Nowadays, elective TP is an established treatment option for large malignant tumors of the pancreas, chronic pancreatitis or multifocal intraductal papillary mucinous neoplasm (IPMN) and it has been increasingly performed at some high-volume pancreas centers (13,23-25). Recent studies have repeatedly reported of comparable rates of morbidity and mortality for elective TP and PD (25-28). With the ascent of modern therapy of diabetes and improved enzymatic formulations even the long-term consequences of TP seem to be under control. Already in 2007, Müller et al. reported of comparable global health status 2 years after TP and PD (26). Other authors found similar outcomes, survival and quality of life after TP and PD $(11,29)$. In the systematic review of Scholten et al., quality of life after TP was only moderately reduced and there was no diabetes related mortality in the studies published after 2005 (30). Respectively, several authors revived the discussion about the feasibility of elective primary TP in selected patients with high-risk pancreas in order to prevent severe POPF and PPH $(11-14,26)$. Notably, Balzano et al. defined exactly the same profile of patients who may benefit from prophylactic TP as in our own series: elderly and obese patients in a poor general condition with a high-risk pancreas (12). Del Chiaro et al. confirmed that TP is still no equal alternative to PD, but may be considered an "adequate alternative technique" in selected high-risk patients (14). In an earlier report by our group, Janot et al. defined technical problems with the construction of $\mathrm{PJ}$ in high-risk pancreatic remnant as one of four typical indications for elective TP (13).

The present study revealed that some selected patients with soft pancreas and small-sized pancreatic duct may really benefit from primary "prophylactic" TP instead of performing PD. Soft pancreatic texture and small pancreatic duct are recognized as the most important risk factors for the development of POPF and are essential components of all current fistula risk scores $(15,22)$. This fact explains the high rate of clinically relevant POPF in the PD group of our study, which included intentionally only selected patients at a substantially increased risk for fistula development, proved by the calculated alternative Fistula Risk Score (a-FRS). Our data also confirmed literature data that obesity corresponds to the presence of a risky pancreatic remnant and to a higher fistula risk score $(15,22,31)$. Interestingly, BMI and ASA score were significantly higher and cardiovascular disease was more often in the TP group. Recently, we showed that exactly these three items represent relevant risk factors for POPF grade $\mathrm{C}$ associated with mortality risk of up to $40 \%$ (31). Since TP and PD patients were strictly matched for all other basic parameters such as diagnosis, gender, age, surgical team and anatomic characteristics of the pancreatic remnant, it seems that perception of obesity and poor general condition may have biased surgeon's decision to perform TP rather than PD. This assumption is supported by the significantly higher a-FRS in the TP group. On the other hand, our subjective belief that the preoperative presence of insulin dependent diabetes affected surgeon's readiness to favor TP was not supported by the current data: patients with TP and PD had similar rates of endocrine and exocrine pancreatic insufficiency before surgery.

Our results differ from other studies reporting longer operative times and increased blood loss with TP compared to PD (26-28). This may be explained by the selection of only high-risk patients, associated with demanding PJ, which construction may sometimes be more timeconsuming than performing completion pancreatectomy itself.

TP and PD resulted in similar rates of mortality and major morbidity; however, there was a higher rate of overall complications after PD due to the higher incidence of mild complications. This fact is surprising having in mind that PD patients were in a better physical condition, had less cardiovascular comorbidities and suffered less obesity. However, it is still remarkable, that in that group of old and sick patients TP was more frequently associated with an uncomplicated postoperative course.

POPF was the most common complication after PD and was naturally absent after TP. This may partially explain the trend towards higher DGE rate after PD, since local inflammation due to POPF is an established risk factor for DGE (16). On the other hand, impaired wound healing was more often after TP, which may be explained by more 
pronounced obesity, hydrocortisone administration in the early postoperative course and development of acute diabetes type $3 \mathrm{c}$.

An important finding of the study refers to the rates of POPF and PPH. As expected, TP eliminated the risk of POPF and postoperative acute pancreatitis completely. Eleven patients developed POPF grade C after PD, leading to a significantly higher rate of $\mathrm{PPH}$ in that group and to 10 completion pancreatectomies with $50 \%$ mortality rate. The question how these 10 patients with emergency TP would have done if they had undergone a prophylactic TP remains open. The fact that bile leak rates were the same, but PPH were more common after PD confirms the hypothesis that pancreatic secretion or the mixture of bile and pancreatic juice is more aggressive to vessels than bile alone (32). Despite significantly higher rate of $\mathrm{PPH}$ after $\mathrm{PD}$, mortality was not increased compared to TP. This may be one positive effect of centralization, allowing lower "failure-torescue" rates at high-volume centers.

Regarding mortality rate our results are consistent with most other large series on TP and PD, especially having in mind the deliberate selection of obese, older patients with a poor general condition and plenty of comorbidities (24-28). Patients with chronic pancreatitis were not included in the study population. This makes our results hardly comparable to other unfiltered and heterogeneous series of consecutive patients. As already published, mortality after PD at our center for the last 15 years was $2.6 \%$ at average (31). In comparison, Krautz et al. reported mortality of $6.5 \%$ at German high-volume centers and up to $12 \%$ at low-volume institutions (33).

A major finding of the present study is the different spectrum of causes of mortality after TP and PD. All 5 deaths in the PD group were triggered by surgical complications. In all of them emergency completion pancreatectomy for severe grade C POPF accompanied by erosion arterial hemorrhage was performed. In contrast, only 2 of 7 lethal outcomes after TP were associated with relevant surgical complications. The rest 5 patients died of progressive respiratory failure due to pneumonia and deteriorated COPD. This detail emphasizes the significant mortality risk posed by the presence of high-risk pancreatic remnant in patients undergoing $\mathrm{PD}$. At the same time, TP may indeed reduce the risk of surgical mortality, but not the overall risk of mortality due to medical complications, which are difficult to prevent in a group of old and polymorbid patients.

A strength of the present study is the relatively high number of patients, treated according to a standardized surgical technique and perioperative management protocol by an experienced team at a dedicated institution. This allowed the exclusion of many important confounders and improved the internal validity of results.

Limitations of the study include its retrospective character and the lack of corresponding large series of prophylactic TP from other centers, which does not allow us to prove the external validity of our findings. Furthermore, long-term effects of TP regarding quality of life and secondary complications due to the apancreatic state were not covered by the present study. These items are included as primary endpoints of an ongoing multicenter trial in Germany with our institution being a participating study center. Other recent studies have focused on quality of life and long-term outcome in detail $(28,29)$.

A limitation of the present study is the lack of direct comparison of TP as the most aggressive available option to methods of pancreatic reconstruction other than the ductto-mucosa technique, which would allow preservation of high-risk pancreata. Pancreatogastrostomy (PG), widely believed to be superior to PJ particularly for patients with soft pancreas, has been studied by eleven randomized controlled trials since 1995 . The largest trials by Keck et al. and by Topal et al. failed to demonstrate lower rates of clinically relevant POPF for PG and even reported a significantly higher rate of PPH for PG $(3,34)$. The Blumgart technique of $\mathrm{PJ}$ has been shown to reduce the rate of POPF in a myriad of retrospective reports. However, recent randomized controlled trials, the largest one being the multicenter PANasta trial from UK failed to find any advantages of the Blumgart technique over the standard Cattel-Warren method with regards to POPF and other postoperative outcomes, as reported earlier this year (35). Both techniques showed a POPF rate of over $25 \%$ in a mixed population of patients with high- and lowrisk pancreatic remnant. Interestingly, in a recent study by Kawakatsu et al. the rate of clinically relevant POPF reached $43 \%$ in a selected cohort of 286 patients with highrisk pancreas. This incidence of POPF was even higher than in our subgroup of PD with duct-to-mucosa PJ, although the Blumgart and Kakita anastomoses were used in combination with externalized pancreatic and biliary stents (36). A wide spectrum of supportive measures such as external and internal stents, wrapping of $\mathrm{PJ}$ with omentum or ligamentum teres patch and application of glues has been found to be of no real value in the prevention of POPF after PD (37). 
The reported high morbidity rates in this study, both after PD and TP, made us rethink our strategy: recently we introduced a novel modified single-loop reconstruction after PD in order to separate bile from pancreatic juice and mitigate the severity of POPF. Combining this method with wrapping of the gastroduodenal stump with a ligamentum teres patch, selective use of octreotide and an enhanced recovery protocol has showed promising results after PD for patients with soft pancreas (32).

The above-mentioned data demonstrate that there is no single safe technique of pancreatic reconstruction after PD which is able to achieve acceptably low rates of POPF in the group of patients with high-risk pancreatic remnant. In this study we compared TP to the standard duct-to-mucosa PJ, which represents the most frequently used technique worldwide.

\section{Conclusions}

In conclusion, the use of prophylactic $\mathrm{TP}$ in cases of high-risk pancreatic remnant deemed unsuitable for a safe anastomosis may be a vital option for the surgeon in order to avoid life-threatening POPF-associated complications in the postoperative period. Since TP remains an aggressive procedure with its own spectrum of major postoperative morbidity and detrimental longterm effects, the decision to perform it should be made by an experienced surgeon, taking into consideration the individual patient's characteristics. Further randomized controlled trials are necessary to establish the best method of pancreatic reconstruction after $\mathrm{PD}$ which can allow the safe preservation of high-risk pancreata and avoid the use of TP.

\section{Acknowledgments}

Funding: None.

\section{Footnote}

Reporting Checklist: The authors have completed the STROBE reporting checklist. Available at http://dx.doi. org/10.21037/gs-20-670

Data Sharing Statement: Available at http://dx.doi. org/10.21037/gs-20-670

Peer Review File: Available at http://dx.doi.org/10.21037/gs-
$20-670$

Conflicts of Interest: All authors have completed the ICMJE uniform disclosure form (available at http://dx.doi. org/10.21037/gs-20-670). The authors have no conflicts of interest to declare.

Ethical Statement: The authors are accountable for all aspects of the work in ensuring that questions related to the accuracy or integrity of any part of the work are appropriately investigated and resolved. This study was approved by the Ethics committee of the Ruhr University Bochum (No. 17-6321) and conducted in accordance with the Helsinki Declaration (as revised in 2013). Informed consent for surgery as well as for data collection and analysis was given by all patients.

Open Access Statement: This is an Open Access article distributed in accordance with the Creative Commons Attribution-NonCommercial-NoDerivs 4.0 International License (CC BY-NC-ND 4.0), which permits the noncommercial replication and distribution of the article with the strict proviso that no changes or edits are made and the original work is properly cited (including links to both the formal publication through the relevant DOI and the license). See: https://creativecommons.org/licenses/by-nc-nd/4.0/.

\section{References}

1. Cameron JL, He J. Two thousand consecutive pancreaticoduodenectomies. J Am Coll Surg 2015;220:530-6.

2. Smits FJ, Verweij ME, Daamen LA, et al. Impact of Complications After Pancreatoduodenectomy on Mortality, Organ Failure, Hospital Stay, and Readmission: Analysis of a Nationwide Audit. Ann Surg 2020. [Epub ahead of print]. doi:10.1097/SLA.0000000000003835.

3. Keck T, Wellner UF, Bahra M, et al. Pancreatogastrostomy versus Pancreatojejunostomy for RECOnstruction after PANCreatoduodenectomy (RECOPANC, DRKS 00000767). Perioperative and longterm results of a multicenter randomized controlled trial. Ann Surg 2016;263:440-9.

4. Eshmuminov D, Schneider MA, Tschuor C, et al. Systematic review and meta-analysis of postoperative pancreatic fistula rates using the updated 2016 International Study Group Pancreatic Fistula definition in patients undergoing pancreatic resection with soft and 
hard pancreatic texture. HPB (Oxford) 2018;20:992-1003.

5. Pedrazzoli S. Pancreatoduodenectomy (PD) and postoperative pancreatic fistula (POPF): A systematic review and analysis of the POPF-related mortality rate in 60,739 patients retrieved from the English literature published between 1990 and 2015. Medicine (Baltimore) 2017;96:e6858.

6. Mackay TM, Smits FJ, Roos D, et al. The risk of not receiving adjuvant chemotherapy after resection of pancreatic ductal adenocarcinoma: a nationwide analysis. HPB (Oxford) 2020;22:233-40.

7. Rockey EW. Total pancreatectomy for carcinoma: case report. Ann Surg 1943;118:603-11.

8. Priestley JT, Comfort MW, Radcliffe J. Total pancreatectomy for hyperinsulinism due to an isletcell adenoma: survival and cure at sixteen months after operation presentation of metabolic studies. Ann Surg 1944;119:211-21.

9. Ross DE. Cancer of the pancreas: a plea for total pancreatectomy. Am J Surg 1954;87:20-33.

10. Porter MR. Carcinoma of the pancreatico-duodenal area: operability and choice of procedure. Ann Surg 1958;148:711-23.

11. Casadei R, Ricci C, Taffurelli G, et al. Is total pancreatectomy as feasible, safe, efficacious, and costeffective as pancreaticoduodenectomy? A single center, prospective, observational study. J Gastrointest Surg 2016;20:1595-607.

12. Balzano G, Maffi P, Nano R, et al. Autologous islet transplantation in patients requiring pancreatectomy: a broader spectrum of indications beyond chronic pancreatitis. Am J Transplant 2016;16:1812-26.

13. Janot MS, Belyaev O, Kersting S, et al. Indications and early outcomes for total pancreatectomy at a high-volume pancreas center. HPB Surg 2010;2010:686702.

14. Del Chiaro M, Rangelova E, Segersvärd R, et al. Are there still indications for total pancreatectomy? Updates Surg 2016;68:257-63.

15. Belyaev O, Munding J, Herzog T, et al. Histomorphological features of the pancreatic remnant as independent risk factors for postoperative pancreatic fistula: a matched-pairs analysis. Pancreatology 2011;11:516-24.

16. Wente MN, Bassi C, Dervenis C, et al. Delayed gastric emptying (DGE) after pancreatic surgery: a suggested definition by the International Study Group of Pancreatic Surgery (ISGPS). Surgery 2007;142:761-8.

17. Bassi C, Marchegiani G, Dervenis C, et al. The 2016 update of the International Study Group (ISGPS) definition and grading of postoperative pancreatic fistula: 11 Years After. Surgery 2017;161:584-91.

18. Wente MN, Veit JA, Bassi C, et al. Post-pancreatectomy haemorrhage (PPH): an International Study Group of Pancreatic Surgery (ISGPS) definition. Surgery 2007;142:20-5.

19. Koch M, Garden OJ, Padbury R, et al. Bile leakage after hepatobiliary and pancreatic surgery: a definition and grading of severity by the International Study Group of Liver Surgery. Surgery 2011;149:680-8.

20. Besselink MG, van Rojssen LB, Bassi C, et al. Definition and classification of chyle leak after pancreatic operation: a consensus statement by the International Study Group on Pancreatic Surgery. Surgery 2017;161:365-72.

21. Dindo D, Demartines N, Clavien PA. Classification of surgical complications: a new proposal with evaluation in a cohort of 6,336 patients and results of a survey. Ann Surg 2004;240:205-13.

22. Mungroop TH, van Rijssen LB, van Klaveren D, et al.; Dutch Pancreatic Cancer Group. Alternative Fistula Risk Score for Pancreatoduodenectomy (a-FRS): design and international external validation. Ann Surg 2019;269:937-43.

23. Almond M, Roberts KJ, Hodson J, et al. Changing indications for a total pancreatectomy: perspectives over a quarter of a century. HPB (Oxford) 2015;17:416-21.

24. Zakaria HM, Stauffer JA, Raimondo M, et al. Total pancreatectomy: short- and long-term outcomes at a high-volume pancreas center. World J Gastrointest Surg 2016;8:634-42.

25. Murphy MM, Knaus WJ 2nd, Ng SC, et al. Total pancreatectomy: a national study. HPB (Oxford) 2009; 11:476-82.

26. Müller MW, Friess H, Kleeff J, et al. Is there still a role for total pancreatectomy? Ann Surg 2007;246:966-74.

27. Petrucciani N, Nigri G, Giannini G, et al. Total pancreatectomy for pancreatic carcinoma. When, why and what are the outcomes? Results of a systematic review. Pancreas 2020;49:175-80.

28. Pulvirenti A, Pea A, Rezaee N, et al. Perioperative outcomes and long-term quality of life after total pancreatectomy. Br J Surg 2019;106:1819-28.

29. Billings BJ, Christein JD, Harmsen WS, et al. Quality-oflife after total pancreatectomy: is it really that bad on longterm follow-up? J Gastrointest Surg 2005;9:1059-66.

30. Scholten L, Stoop TF, Del Chiaro M, et al. Systematic review of functional outcome and quality of life after total pancreatectomy. Br J Surg 2019;106:1735-46. 
31. Luu AM, Krasemann L, Fahlbusch T, et al. Facing the surgeon's nightmare: incidence and management of postoperative pancreatic fistulas grade $\mathrm{C}$ after pancreaticoduodenectomy based on the updated definition of the International Study Group on Pancreatic Surgery (ISGPS). J Hepatobiliary Pancreat Sci 2020;27:171-81.

32. Aghalarov I, Herzog T, Uhl W, et al. A modified singleloop reconstruction after pancreaticoduodenectomy reduces severity of postoperative pancreatic fistula in highrisk patients. HPB (Oxford) 2018;20:676-83.

33. Krautz C, Nimptsch U, Weber GF, et al. Effect of hospital volume on in-hospital morbidity and mortality following pancreatic surgery in Germany. Ann Surg 2018;267:411-7.

34. Topal B, Fieuws S, Aerts R, et al. Belgian Section of Hepatobiliary and Pancreatic Surgery.

Cite this article as: Luu AM, Olchanetski B, Herzog T, Tannapfel A, Uhl W, Belyaev O. Is primary total pancreatectomy in patients with high-risk pancreatic remnant justified and preferable to pancreaticoduodenectomy? a matched-pairs analysis of 200 patients. Gland Surg 2021;10(2):618-628. doi: 10.21037/gs-20-670
Pancreaticojejunostomy versus pancreaticogastrostomy reconstruction after pancreaticoduodenectomy for pancreatic or periampullary tumours: a multicentre randomised trial. Lancet Oncol 2013;14:655-62.

35. Halloran C, Neoptolemos JP, Platt K, et al. PANasta Trial: Cattell Warren versus Blumgart techniques of pancreaticojejunostomy following pancreato-duodenectomy-A double-blinded multi-centered trial, trial results. J Clin Oncol 2020;38:abstr 4619.

36. Kawakatsu S, Inoue Y, Mise Y, et al. Comparison of pancreatojejunostomy techniques in patients with a soft pancreas: Kakita anastomosis and Blumgart anastomosis. BMC Surg 2018;18:88.

37. Belyaev O, Uhl W. Evidence-based supportive measures to secure pancreatic anastomoses. Chirurg 2017;88:30-5. 